\title{
Restoration of Cytoskeleton Homeostasis After Gigaxonin Gene Transfer for Giant Axonal Neuropathy
}

\author{
Silke Mussche, Bart Devreese, ${ }^{2}$ Sahana Nagabhushan Kalburgi, ${ }^{3}$ Lavanya Bachaboina, ${ }^{3, *}$ \\ Jonathan C. Fox, ${ }^{3}$ Hung-Jui Shih, ${ }^{3}$ Rudy Van Coster, ${ }^{1}$ R. Jude Samulski, ${ }^{3}$ and Steven J. Gray ${ }^{3}$
}

\begin{abstract}
Giant axonal neuropathy (GAN) is caused by loss of function of the gigaxonin protein. On a cellular level GAN is characterized by intermediate filament (IF) aggregation, leading to a progressive and fatal peripheral neuropathy in humans. This study sought to determine if re-introduction of the GAN gene into GAN-deficient cells and mice would restore proper cytoskeleton IF homeostasis. Treatment of primary skin fibroblast cultures from three different GAN patients with an adeno-associated virus type 2 (AAV2) vector containing a normal human GAN transgene significantly reduced the number of cells displaying vimentin IF aggregates. A proteomic analysis of these treated cells was also performed, wherein the abundance of 32 of 780 identified proteins significantly changed in response to gigaxonin gene transfer. While 29 of these responding proteins have not been directly described in association with gigaxonin, three were previously identified as being disregulated in GAN and were now shifted toward normal levels. To assess the potential application of this approach in vivo and eventually in humans, GAN mice received an intracisternal injection of an AAV9/GAN vector to globally deliver the GAN gene to the brainstem and spinal cord. The treated mice showed a nearly complete clearance of peripherin IF accumulations at 3 weeks post-injection. These studies demonstrate that gigaxonin gene transfer can reverse the cellular IF aggregate pathology associated with GAN.
\end{abstract}

\section{Introduction}

G IANT AXONAL NEUROPATHY (GAN, OMIM \#256850) is a rare chronic neurodegenerative disease characterized by enlarged axons with disordered microtubules and intermediate filaments, which is fatal by the third decade of life. The disease pathology is due to homozygous loss-of-function mutations in the GAN gene, which encodes the protein gigaxonin. Onset of symptoms is usually 3-4 years of age, with a slightly awkward gait. By the end of the second decade of life, patients normally are wheelchair bound with limited use of the arms and little to no use of their legs. Death normally occurs in the second or third decade of life. Peripheral nerve biopsies from humans show enlarged axons with densely packed and disorganized microtubules and intermediate filaments (IFs) (Demir et al., 2005; Nalini et al., 2008). The dysfunction and degeneration of peripheral nerves is attributed to this pathology. These same studies showed white matter abnormalities in the brain by magnetic resonance imaging, indicating an involvement of the central nervous system. This peripheral nerve pathology (enlarged axons densely filled with neurofilaments) serves as a basis for diagnosis, which is then confirmed by sequencing the GAN gene. There are no statistics on the incidence of the disease, but it is considered extremely rare.

Giant axonal neuropathy was characterized over 25 years ago as an inborn error in intermediate filament organization (Pena et al. 1983), which was later found to be from mutations in the gigaxonin gene and resulting loss of functional gigaxonin protein (Bomont et al., 2000). More than $40 \mathrm{mu}$ tations have been identified in GAN patients, including deletions, insertions, missense and nonsense mutations, which lead to loss of function of gigaxonin (Bomont et al., 2000, 2003; Bruno et al., 2004; Demir et al., 2005; Houlden et al., 2007; Koop et al., 2007; Kuhlenbäumer et al., 2002; Leung et al., 2007). Gigaxonin is a broadly expressed Cul3 ubiquitin ligase adaptor protein, normally present at extremely low levels throughout the brain (Cleveland et al., 2009). Known direct targets of gigaxonin are MAP8, MAP1B, and TCB-C, which are involved with IF regulation (Allen et al., 2005;

\footnotetext{
${ }^{1}$ Department of Pediatrics, Division of Pediatric Neurology and Metabolism, Ghent University Hospital, Ghent 9000, Belgium.

${ }^{2}$ Laboratory for Protein Biochemistry and Biomolecular Engineering, Ghent University, Ghent 9000, Belgium.

${ }^{3}$ Gene Therapy Center, University of North Carolina at Chapel Hill, Chapel Hill, NC 27599.

*Current address: USA Mitchell Cancer Institute, 1660 Springhill Avenue, Mobile, AL 36604.
} 
Ding et al., 2002, 2006; Wang et al., 2005). In the absence of gigaxonin, the regulation of IFs is disrupted, leading to increased IFs that can form inclusion bodies. The composition of these inclusions varies based on the specific IF composition in any given cell type. Vimentin, peripherin, neurofilaments (NF-H, NF-M, NF-L), alpha-internexin, GFAP, nestin, desmin, and keratin are IFs known to accumulate in mice and/or humans lacking gigaxonin (Berg et al., 1972; Dequen et al., 2008; Pena et al., 1983). Abnormal aggregates of keratin in hair are likely responsible for the typical curled hair observed in most patients (Bomont and Koenig, 2003; Pena et al., 1983; Prineas et al., 1976). The vimentin aggregates in fibroblasts are located in the perinuclear space close to the microtubule organizing center (Bomont and Koenig, 2003). Gigaxonin is also a substrate for ubiquitination and degradation by its own Cul3 ubiquitin ligase complex, providing a level of self-regulation at the protein level (Zhang et al., 2005).

Unfortunately, the underlying function(s) of gigaxonin and the mechanism by which the protein regulates IFs is poorly understood. The structural/functional domains of gigaxonin include an N-terminal BTB domain (BR-C, ttk, and bab), a centrally located conserved BACK domain, and six kelch motifs in the C-terminal part. Gigaxonin has a suspected role as a substrate-specific adaptor for the BCR (BTB/ Kelch protein-cullin-3-RING box protein1) E3 ubiquitin ligase complex (Furukawa et al., 2003). As such an adaptor, it may facilitate proteasome targeting and degradation of various substrate proteins such as MAP1B, MAP8, and TBC-B via interactions with its C-terminal kelch domains (Ding et al., 2002, 2006; Wang et al., 2005). It has been postulated that these substrates accumulate in the absence of gigaxonin, and this hypothesis was partly confirmed in GAN knock-out (KO) mice (Allen et al., 2005; Ding et al., 2006; Wang et al., 2005) but not in human cells (Cleveland et al., 2009). However, no direct link has been established to explain how this leads to disregulation of IFs.

Conceptually, the unbalanced regulation of IFs could be corrected by re-introducing gigaxonin. In such a scenario, it is also conceivable that restoring normal IF regulation could allow cells to resolve IF inclusions and reverse existing pathology. Carriers of mutant gigaxonin alleles (humans and mice) express approximately 50\% levels of gigaxonin and are phenotypically and pathologically normal, suggesting that restoration of lower-than-normal gigaxonin levels could still be efficacious. While gross overexpression of gigaxonin has a conceptual risk for toxicity, the protein-level autoregulation of gigaxonin abundance should correct for mild overexpression. Therefore, a broad therapeutic index of gigaxonin expression levels is expected. For these reasons, we propose that GAN is an excellent candidate for a gene replacement therapy. This study sought to determine if re-introduction of gigaxonin into GAN-deficient cells would restore proper cytoskeleton IF homeostasis, reversing the cellular disease phenotype of IF aggregation. In this study, skin fibroblast cultures from three different GAN patients were transduced with an adeno-associated virus type 2 (AAV2) vector containing a normal human GAN transgene or a green fluorescent protein (GFP) transgene as a control. Reduction in vimentin aggregation following viral delivery was scored microscopically. To further characterize the global intracellular changes associated with gigaxonin re-introduction, the proteome of these cells was analyzed after viral delivery, identifying and measuring the relative abundance of proteins using a differential proteomics method based on the iTRAQ strategy in combination with two-dimensional liquid chromatography mass spectrometry (2D-LC-MS) on a MALDI TOFTOF MS platform. Finally, to assess the potential application of this approach in vivo and eventually in humans, GAN-KO mice were injected with an AAV9/GAN vector and scored for reduction of the neuronal IF peripherin in the brainstem and spinal cord. This study sought to understand intracellular changes that occur in response to the re-introduction of gigaxonin into GAN-null cells, both in vitro and in vivo.

\section{Methods}

\section{Patients fibroblast cultures}

Primary skin fibroblasts were maintained in MEM-alpha (Gibco no. 12561) containing penicillin and streptomycin with $10 \%$ fetal bovine serum (FBS), at $5 \% \mathrm{CO}_{2}$ and $37^{\circ} \mathrm{C}$. P1 has a homozygous missense mutation (p. Ala49Glu), P2 has heterozygous mutations (p. S52G and C393X), and P3 has a homozygous deletion (c.1647-8680_*258del, encompassing exons 10 and 11). These patients were all clinically verified to be GAN patients with typical symptoms. P2 was described previously (Bomont et al., 2000), and an Ala49Thr (similar to P1) has been described (Bruno et al., 2004). The normal human fibroblast line was transformed with hTERT.

\section{Vector preparation}

rAAV vectors were produced using a triple-transfection method in HEK293 cells as previously described (Gray et al., 2011a). Highly pure recombinant virus was recovered using sequential step and continuous $\mathrm{CsCl}$ gradients, then the peak fractions were dialyzed in phosphate-buffered saline (PBS) containing 5\% D-sorbitol. Viral titers were determined by dot blot or quantitative polymerase chain reaction (qPCR) as described (Gray et al., 2011a). The recombinant vectors in these studies were single-stranded AAV using AAV2 inverted terminal repeats, packaged in either an AAV2 or AAV9 capsid. The CMV-GAN construct used a cytomegalovirus (CMV) enhancer/promoter, myc-tagged gigaxonin coding sequence, and SV40 polyA. The GFP construct used the same CMV enhancer/promoter, enhanced GFP coding sequence, and SV40 polyA.

\section{Vector treatments in vitro, immunocytochemistry, and vimetin aggregate scoring}

For proteomic analysis, cells were grown in $10 \mathrm{~cm}$ plates. Cells were seeded $2.5 \times 10^{5}$ per plate, in triplicate to three plates. After 1 day, $5 \times 10^{10}$ vector genomes $(\mathrm{vg})$ of either ssAAV2/CMV-GAN or ssAAV2/CMV-GFP were added to each plate. Three days post-infection, cells were harvested with trypsin, washed with PBS, and cell pellets were frozen at $-80^{\circ} \mathrm{C}$ until use.

For immunocytochemistry, 10,000 cells per well were seeded onto poly-L-lysine coated coverslips (BD no. 354085) in 24-well plates on day 0 . On day $1,5 \times 10^{9} \mathrm{vg}$ of AAV2 vector, or a corresponding amount of PBS containing $5 \%$ sorbitol, was added per well. On day 2 the media was changed. On days 4-5 (3-4 days post-infection), cells were washed with PBS and fixed with $4 \%$ paraformaldehyde for $10 \mathrm{~min}$ at room temperature. Cells were then washed with 
PBS and incubated twice for $10 \mathrm{~min}$ at room temperature with PBS containing $0.1 \%$ Triton-X100. Blocking was done for $1 \mathrm{hr}$ at room temperature with PBS containing 3\% goat serum and $0.1 \%$ Triton-X100. Primary antibody incubation was for $1 \mathrm{hr}$ at room temperature with PBS containing 3\% goat serum, $0.1 \%$ Triton-X100, 1:1000 dilution of mouse antivimentin (Millipore no. CBL202), and 1:500 dilution of rabbit anti-GFP (Millipore no. 3080). After three washings with PBS containing $0.1 \%$ Triton-X100, secondary antibody incubation was for $1 \mathrm{hr}$ in PBS containing 3\% goat serum, $0.1 \%$ TritonX100, goat anti-mouse Alexa594 (Invitrogen no. A11032), and goat anti-rabbit Alexa488 (Invitrogen no. A11008). After washing twice with PBS, coverslips were mounted onto slides using ProLong Gold Antifade mounting media with 4',6-diamidino-2-phenylindole (DAPI; Invitrogen no. P-36931). The presence of vimentin aggregates were scored visually, with $>500$ cells typically assessed per coverslip.

\section{qPCR analysis of vector genomes and gigaxonin mRNA expression}

RNA was recovered from the cells using the RNA Mini Plus kit (Qiagen); tissue lysis was performed with the TissueLyser (Qiagen), and automated purification was carried out on a Qiacube (Qiagen). RNA was treated to remove any residual genomic DNA as follows: RNA was incubated for $10 \mathrm{~min}$ at $37^{\circ} \mathrm{C}$ with $20 \mathrm{U}$ Protector RNase Inhibitor (Roche), $1 \times$ DNase I reaction buffer (NEB) and $2 \mathrm{U}$ of DNase I (NEB). This was followed by an additional incubation of $10 \mathrm{~min}$ at $75^{\circ} \mathrm{C}$ with EDTA added to a final concentration of $2.5 \mathrm{mM}$ for DNaseI inactivation. cDNA was synthesized from the purified RNA using the Transcriptor First Strand cDNA Synthesis kit (Roche), following the manufacturer's instructions, then used directly as template for reverse transcription (RT)-qPCR. RTqPCR amplifications and analysis were carried out on a Roche Lightcycler 480, following the manufacturer's instructions and as described (Gray et al., 2010). All primer sets used SyBR green for quantitation except for the GAPDH primer, which used a primer and hydrolysis probe combination.

To quantify the myc-tagged gigaxonin transgene separate from the endogenous gigaxonin, primers were designed so that the upstream primer annealed to the myc tag. An internal set of gigaxonin primers were also used that would equally recognize the endogenous and transgene gigaxonin. qPCR of cDNA samples was used to determine the absolute numbers of myc-gigaxonin, total gigaxonin, and GAPDH transcripts per unit volume in each sample. Transcript quantification is reported as the absolute number of gigaxonin transcripts per microliter divided by the absolute number of actin transcripts per microliter in the sample. Primers for myc-tagged human gigaxonin were as follows: FORWARD: 5'-tgatcagcgaggaggacctgg-3'; REVERSE: 5'gttgatccatcatcttttggagg- $3^{\prime}$. Internal gigaxonin primers recognizing the endogenous and transgene gigaxonin were as follows: FORWARD: 5'-ggttatgagagagatcctgga-3'; REVERSE: 5'-cgtgatggaggcagtaatgta-3'. The human GAPDH reactions use the following primers and probe: FORWARD: $5^{\prime}$-agcca catcgctcagacac-3'; REVERSE: 5'-gcccaatacgaccaaatcc-3'; PROBE: UPL\#60 (Roche cat no. 04688589001). A melting curve analysis was performed at the end of each SyBR green qPCR run, following the manufacturer's instructions, to verify the purity of the PCR product.

\section{ITRAQ reagent labeling and peptide separation}

Proteins were extracted by resuspending each cell pellet in $200 \mu \mathrm{L}$ of lysis buffer, containing $0.05 M$ triethyl ammonium bicarbonate, $7 \mathrm{M}$ urea, $2 \mathrm{M}$ thiourea, and protease inhibitor cocktail (Roche). The cells were sonicated, and the supernatant was collected after centrifugation at $13,000 \times g$ for $20 \mathrm{~min}$ at $4^{\circ} \mathrm{C}$. An acetone precipitation was performed prior to the protein concentrations measurement by the Bradford protein assay (Thermo Scientific). A summary of the experimental workflow is shown in Supplementary Figure S2 (Supplementary Data are available online at www.liebertpub.com/ hum). Equal amounts of protein $(25 \mu \mathrm{g})$ from each cell lysate were used. The iTRAQ labeling was performed according to the manufacturer's protocol using solvents included in the kit (AB SCIEX). The six peptide samples were added to the different labels of the 8-plex iTRAQ kit (AB SCIEX) as follows: patient 1 (P1), 113 (GAN) and 114 (GFP); patient 2 (P2), 115 (GAN) and 116 (GFP); and patient 3 (P3), 117 (GAN) and 118 (GFP). The labels were added in excess to obtain full labeling. Labeling was confirmed for all samples before combining the samples. After combining the six iTRAQ-labeled samples, the mixture was vacuum dried and dissolved in $20 \mathrm{mM}$ $\mathrm{HCOONH}_{4}, 2 \%$ acetonitrile (ACN) at $\mathrm{pH} 10$.

The labeled peptide mixture was separated on an Ettan liquid chromatography system (GE Healthcare), using a Luna C18 column $(2.0 \mathrm{~mm}$ i.d. $\times 150 \mathrm{~mm}$ length $\times 5 \mu \mathrm{m}, 100 \AA$ column; Phenomenex), as described by Mussche et al. (2012). The buffer compositions were $20 \mathrm{mM} \mathrm{HCOONH}_{4}, 2 \% \mathrm{ACN}$ at $\mathrm{pH}$ 10 for buffer A and $20 \mathrm{mM} \mathrm{HCOONH}_{4}, 80 \% \mathrm{ACN}$ at $\mathrm{pH} 10$ for buffer $B$. The flow rate was $0.2 \mathrm{~mL} / \mathrm{min}$, and the elution gradient was as follows: $7 \mathrm{~min} 100 \% \mathrm{~A}, 60 \mathrm{~min} 0 \%-50 \% \mathrm{~B}, 15 \mathrm{~min}$ $50 \%-100 \%$ B, $10 \mathrm{~min} 100 \%$ B, and 3 min $100 \%$ A. Fractions were collected every minute. After drying, the fractions were redissolved in $0.1 \%$ formic acid for nano-LC/MS analysis.

\section{Nano-LC/MS analysis}

Off-line LC-MS was done as described by Mussche et al. (2012). Briefly, analytical reversed phase separation of the labeled peptide mixture was performed on an Ultimate Plus Dual-gradient Capillary/Nano-LC system (Dionex-LC Packings) using a C18 column $(75 \mu \mathrm{m}$ i.d. $\times 150 \mathrm{~mm}$ length, $3 \mu \mathrm{m}$, $100 \AA$; Dionex) at a flow rate of $250 \mathrm{~nL} / \mathrm{min}$. The mobile phases were $5 \%$ ACN/0.05\% trifluoroacetic acid (TFA), $\mathrm{pH} 3$ for buffer A and 80\% ACN/0.05\% TFA, pH 3 for buffer B. Peptides were resolved by gradient elution using a gradient of $0 \%-50 \%$ buffer B over $25 \mathrm{~min}, 50 \%-100 \%$ buffer B over $10 \mathrm{~min}$, $5 \mathrm{~min}$ of $100 \%$ buffer B, and $10 \mathrm{~min}$ of $100 \%$ buffer A. Fractions were collected every $30 \mathrm{sec}$ directly on a MALDI target plate. The MALDI plates were analyzed by a $4800+$ MALDI-TOFTOF Proteomics Analyzer (AB SCIEX). A plate calibration was performed using the 4700 calibration mixture with a mass tolerance of $0.5 \mathrm{~m} / \mathrm{z}$. The MSMS mode was calibrated with a mass tolerance of $0.1 \mathrm{~m} / z$. Additionally, MS spectra were internally calibrated with Glu-fibrinopeptide $(1552.62 \mathrm{~m} / \mathrm{z})$. MS data were acquired in the reflector positive mode over a mass range of 900-3500 m/z, by accumulation of 1000 laser shots per spectrum. The eight highest precursor masses in each MS spectrum with an $\mathrm{S} / \mathrm{N}$ threshold of minimum 50, were selected for fragmentation, and the fraction-to-fraction mass tolerance was set to $200 \mathrm{ppm}$. MS/MS data were acquired with 2000 laser shots per spectrum, using $1 \mathrm{keV}$ collision 
energy and air as the collision gas. Each plate was analyzed twice by reinterpretation of the MS spectra with a method containing an exclusion list from initial MS/MS data to avoid analyzing peptides more than once.

\section{Database searching and data analysis}

Data analysis was performed with the software package Mascot Daemon (Perkins et al., 1999; version 2.3, Matrix science). All MS/MS spectra were processed against the UniProtKB/SwissProt Homo sapiens database. Search parameters included iTRAQ labeling at the N-terminus and lysine residues, cysteine modification by methyl methanethiosulfonate, digestion by trypsin (including two missed cleavages allowed), and methionine oxidation as a variable modification. Normalization of the ratios was performed by the median ratio. A significance threshold of $p<0.05$ was used for peptide identification and an ion score cutoff of 30. Only unique peptides were selected for quantification, and protein ratios were calculated by the weighted average of the peptide ratios. Using the build in decoy function, searching a concatenated database containing both forward and shuffled decoy sequences, allowed estimation of the false discovery rate (Reidegeld et al., 2008). The ratio of GAN/GFP was considered in the three patients and accepted as significant regulated when the ratios of all three patients samples showed a significant value at the 95\% confidence level $(p<0.05)$.

The function, interactions and localization of the differential regulated proteins were studied based on information in databases such as Uniprot, neXtProt, and STRING (Snel et al., 2000), only considering experimentally confirmed interactions.

\section{Western blotting of fibroblast samples}

For validation of the proteins found by iTRAQ, fibroblast protein extracts of each sample (proteins sonicated in lysis buffer) were separated by tricine-SDS-PAGE on a $16.5 \%$ polyacrylamide gel. Proteins were transferred by electroblotting $(3 \mathrm{hr}$ at $60 \mathrm{~V})$ onto a nitrocellulose membrane (Invitrogen). Immunoblotting was carried out with primary antibodies against the galectin-1 (ab25138, Abcam), PARK7/ DJ1 (Abcam ab18257), and $\beta$-actin (Abcam). The intensity of the protein bands was quantified using the Quantity One imaging software (Bio-Rad).

\section{Mouse studies}

All investigations were approved by the University of North Carolina-Chapel Hill Institutional Animal Care and Use Committee. Ten microliters of ssAAV9/CMV-GFP $\left(8 \times 10^{10} \mathrm{vg}\right)$ or ssAAV9/CMV-GAN $\left(7.2 \times 10^{10} \mathrm{vg}\right)$ vector were injected into the cisterna magna. The AAV9/GFP injections were done in 19-20 month-old heterozygous GAN mice, and the ssAAV9/GAN injections were done in $\mathrm{KO}$ $\mathrm{GAN}^{\Delta e x 1}$ (Dequen et al., 2008) or GAN ${ }^{\Delta e x 3-5}$ (Ding et al., 2006) mice. Three weeks following vector injection, each mouse was sacrificed and transcardially perfused with PBS or PBS containing $1 \mathrm{U} / \mathrm{mL}$ heparin (Abraxis).

\section{Immunohistochemistry to detect peripherin aggregates}

After $48 \mathrm{hr}$ of fixation in freshly made PBS with $4 \%$ paraformaldehyde, the entire brain and portions of the cer- vical and lumbar spinal cords were sectioned at $40 \mu \mathrm{m}$ using a Leica vibrating microtome. Every fifth section was processed for immunohistochemistry (IHC). Samples were incubated for $1 \mathrm{hr}$ at room temperature in blocking solution ( $10 \%$ goat serum, $0.1 \%$ triton $X-100,1 \times$ PBS), then incubated $48-72 \mathrm{hr}$ at $4{ }^{\circ} \mathrm{C}$ in primary antibody solution ( $3 \%$ goat serum, $0.1 \%$ triton $\mathrm{X}-100,1 \times$ PBS, and primary antibody). Primary antibodies were as follows: rabbit anti-GFP (Millipore no. AB3080, 1:500), rabbit anti-peripherin (Millipore no. AB1530, 1:500), mouse anti-neurofilament heavy chain (NF-H, Covance no. SMI-32P, 1:1000). After washing three times in PBS, secondary amplification was performed using a VectaStain ABC Elite Kit (Vector Labs, no. PK-6101 for rabbit and PK6102 for mouse, as appropriate) with 3,3'-diaminobenzidine tetrachloride (DAB; Polysciences, Inc. no. 04008) substrate and nickel-cobalt intensification of the reaction product.

DAB-processed brain sections were digitized using a ScanScope slide scanner (Aperio Technologies). Virtual slides were viewed using ImageScope software package (v. 10.0; Aperio Technologies), which allowed sections to be viewed at equivalent magnifications.

\section{Western blot of mouse tissue}

To validate expression of the gigaxonin transgene following intracisternal vector delivery, a small portion of the caudal brainstem was homogenized and subjected to SDS-PAGE, then blotted. The myc-tagged gigaxonin band was detected with a goat anti-gigaxonin antibody (N-18, Santa Cruz no. $49688,1: 300$ ) or a mouse anti-myc antibody (Millipore no. 05724, 1:1000).

\section{Results}

\section{Reduction of vimentin aggregates following gigaxonin gene transfer}

The first part of this study sought to investigate the effects of AAV/gigaxonin gene transfer into GAN patient fibroblasts, in terms of the IF organization and potentially broader effects on the proteome (Fig. 1). Dermal fibroblasts taken from human GAN patients show cytoskeletal disorganization and aggregation of the IF vimentin when grown in culture, and these accumulations may be enhanced by culturing them in low serum media $(0.1 \%$ FBS) or growing cells to confluence for 3 days (Bomont and Koenig, 2003). We have observed that when these cells are grown on poly-Llysine coated coverslips the accumulations are enhanced even in 10\% FBS-containing media. Untransformed fibroblasts from three GAN patients and a normal human fibroblast line were seeded, then infected the next day with AAV2 vectors (CMV-GFP, CMV-GAN, or PBS "mock") at an multiplicity of infection (MOI) of $5 \times 10^{4} \mathrm{vg} / \mathrm{cell}$. The media was changed $24 \mathrm{hr}$ after infection and then the cells were grown in complete media (10\% FBS) for an additional 2-3 days (3-4 days post-infection). Cells were then fixed and immunolabeled for vimentin, and the percentage of vimentin aggregate-containing cells were scored.

Approximately $53 \%$ of the fibroblasts were transduced (average of nine trials, range $30 \%$ to $71 \%$ ), as gauged by the number of cells GFP-positive in the AAV2/GFP control group. No suitable anti-gigaxonin antibodies were available to immuno-label for cells expressing the gigaxonin transgene, 


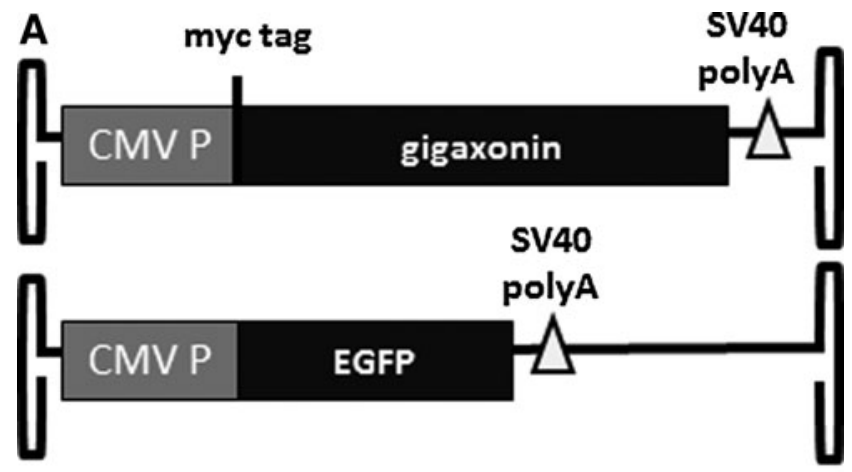

B

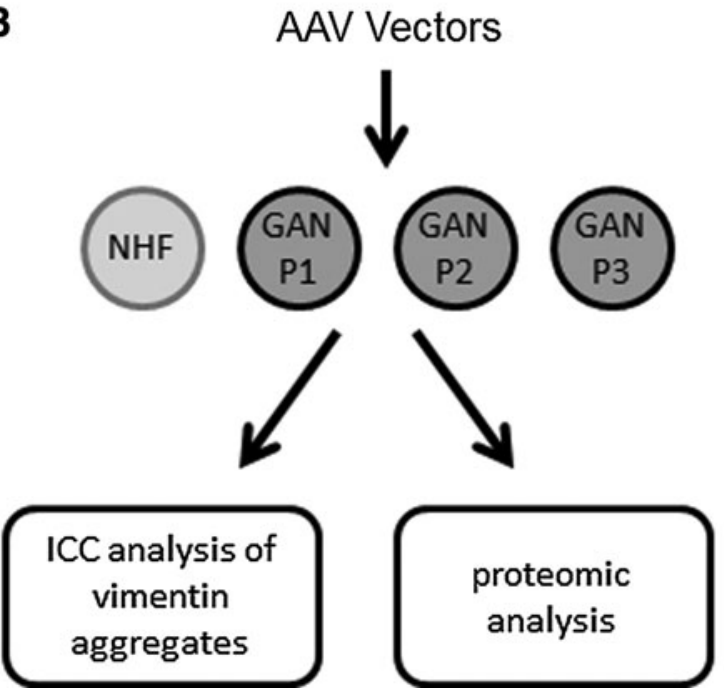

FIG. 1. Study design. (A) Diagram of the packaged singlestranded rAAV genomes used in these studies. (B) Workflow for in vitro experiments using normal (NHF) or GAN patient (GAN) fibroblasts. rAAV, recombinant adeno-associated virus; GAN, giant axonal neuropathy.

and immunocytochemistry against the myc tag also failed to detect the gigaxonin protein. Treatment with the AAV2/GAN vector resulted in a significant reduction in the percentage of cells containing vimentin aggregates, as compared to the mock-infected and AAV2/GFP-infected cells (Fig. 2). Analysis of the mRNA from the cells by RT-qPCR indicated a 3.8-, 1.1-, and 12.4-fold (P1, P2, and $\mathrm{P} 3$ respectively) average increase in gigaxonin mRNA in GAN-treated patient cells versus mocktreated patient cells. The amount of gigaxonin mRNA seen in mock-treated patient cells was equivalent when comparing P1 and P2 to normal human fibroblasts, but the P3 mRNA levels were lower. This is understandable since P1 and P2 had missense mutations that were unlikely to affect the mRNA expression and stability, whereas P3 had a homozygous deletion at the 3 ' end of the gene. The overall average amount of gigaxonin mRNA in GAN-treated cells was 6.2-, 2.6-, and 5.7fold (P1, P2, and P3 respectively) above that detected in normal human fibroblasts. These results indicated that gigaxonin was being mildly overexpressed at 3 days post-infection in this experiment.

There was a small decrease in the overall number of fibroblasts following the AAV2/GAN treatment, suggesting the possibility of overexpression-related toxicity, but it isn't clear if this was due to the gigaxonin gene or high doses of
AAV used ( $p=0.03$ compared to mock treatment and $p=0.69$ compared to GFP treatment, Supplementary Fig. S1). No vimentin aggregates were observed in any of the normal human fibroblasts, and no obvious change in vimentin was observed in the normal human fibroblasts following AAV2/GAN treatment. These results indicate that gigaxonin can function to reduce vimentin IF aggregations and restore normal vimentin organization within 3-4 days of infection.

\section{Proteome analysis}

Currently, there is an incomplete understanding of the cellular function of gigaxonin, and gaps of knowledge exist regarding both its direct binding partners and its downstream targets. To assess the more global consequence of gigaxonin re-introduction into GAN patient fibroblasts, we undertook a proteomic analysis of the cells following AAV2/ GAN or AAV2/GFP gene transfer. Protein extracts from the total cell lysate from six cultured fibroblast cell line samples (three GAN-infected and three GFP-infected patient cultures) were trypsinized and iTRAQ-labeled, followed by reversed phase (RP)-RP LC and MALDI-TOFTOF MS/MS analysis for identification and quantitation. All RP-LC MALDI-TOFTOF runs were searched against the UniProtKB Homo sapiens database, resulting in identification of 14,460 peptides leading to 780 proteins. Proteins with all six iTRAQ labels were considered in the final list. The iTRAQ protein ratios reported here are weighted averages calculated from the ratios of the individual peptides determined for each protein with the accompanying SD and $p$ value. From the total of 780 proteins identified with $>95 \%$ confidence, 31 proteins were found to be significantly regulated $(p<0.05)$ between GANand GFP-transfected cells in all three patients (Table 1). Changes seen between different samples will not be due to any AAV-specific effects since each AAV2/GAN-treated culture was compared with an AAV2/GFP-treated culture from the same patient.

The differentially regulated proteins were further analyzed according to their function, interaction and localization, based on information in databases such as UniProt, neXtProt, and STRING (Snel et al., 2000). Some possible interesting interactions with components of the ubiquitin-proteosome system are listed in Supplementary Table S1. A set of proteins previously described to be differentially regulated in GAN patients versus controls was found not altered in response to the AAV2/GAN treatment (Supplementary Table S2). A list of all proteins detected is provided as Supplementary Table S3. Interestingly, we found no change in the overall levels of vimentin in response to the AAV2/GAN treatment.

Western blot analysis was performed to validate results obtained from the proteome analysis. Galectin-1 and DJ-1 were selected, as according to the iTRAQ results these were significantly down-regulated following gigaxonin gene transfer, and galectin-1 was previously shown to be overexpressed in GAN patients compared to controls (Mussche et al., 2012). This means that this is probably an important player in GAN. Even though the degree of reduction detected in the proteomic analysis was modest (0.62-0.88 fold compared to the GFP treatment), the Western blot results confirmed the down regulation of galectin-1 and DJ-1 in the transduced fibroblasts (Fig. 3). 

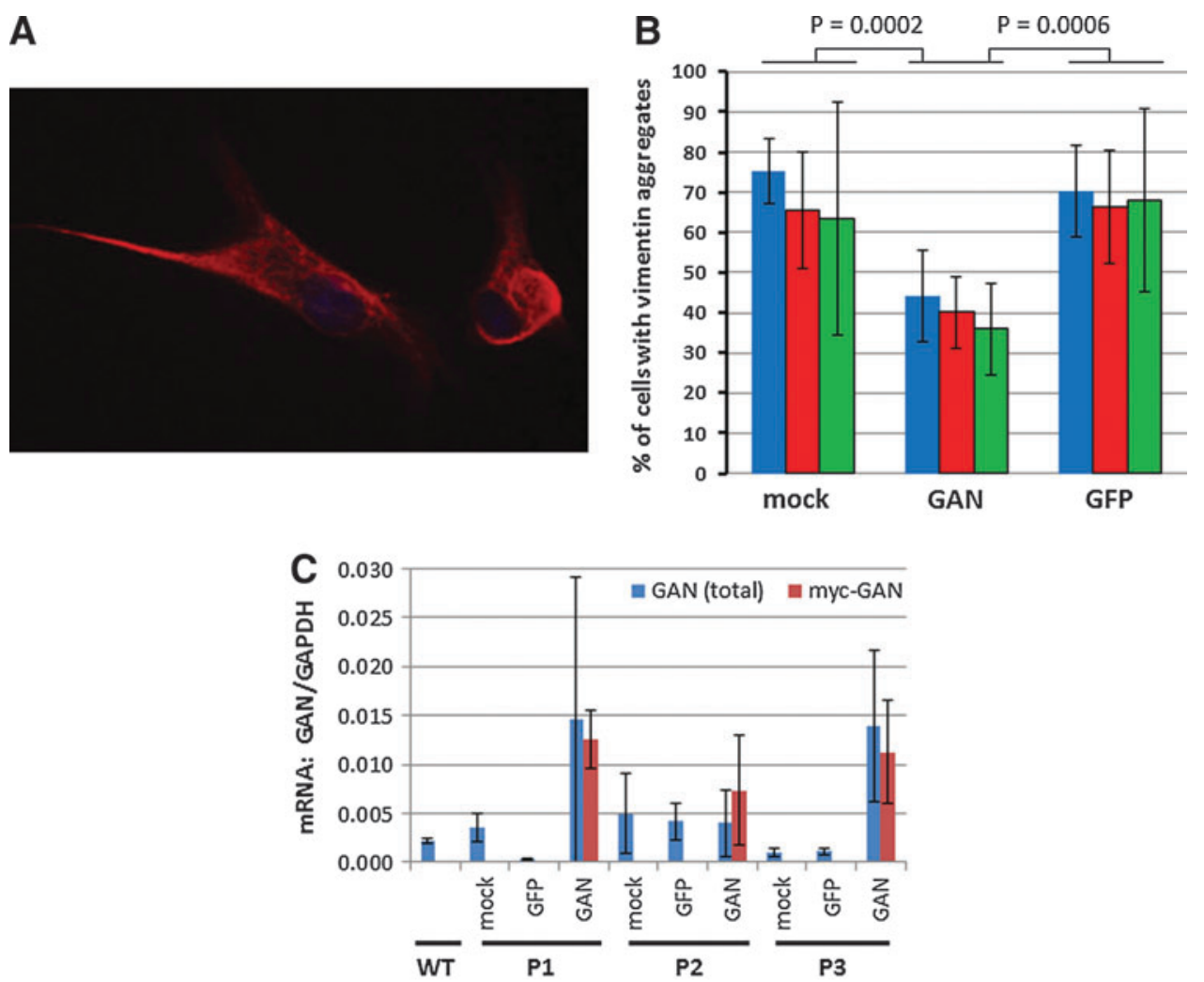

FIG. 2. Reduction of vimentin aggregates after AAV/gigaxonin gene transfer. Cultured GAN patient fibroblasts were treated with in parallel with vehicle buffer (mock), AAV2/GAN vector (GAN), or AAV/GFP vector (GFP), then fixed and immunostained for vimentin after 3-4 days. (A) Representative images of cells with (right) or without (left) the vimentin aggregate phenotype. Vimentin is red and 4',6-diamidino-2-phenylindole (DAPI) is blue. (B) Vimentin aggregates were manually scored, and the average percentage of cells containing vimentin aggregates plotted. Three different lines were used: P1 ( $n=5$, blue bars), P3 ( $n=4$, red bars), and P2 ( $n=2$, green bars). (C) An additional set of cells was collected to assess relative level of gigaxonin expression by mRNA analysis after 3 days. "GAN (total)" used primers that recognize endogenous and exogenous gigaxonin, and "myc-GAN" used primers that only recognize the exogenously delivered gigaxonin. Error bars $=S E M ; p$ values calculated using a Student's $t$ test, one-tailed, unequal variance.

\section{In vivo rescue of peripherin IF aggregates following central nervous system AAV9/GAN gene transfer}

To assess the possible translational potential of an AAV/ GAN vector, a similar experiment to the in vitro vimentin IF rescue was undertaken in vivo. There are three $\mathrm{KO}$ mouse models described for GAN (Dequen et al., 2008; Ding et al., 2006; Ganay et al., 2011). The first was described as recapitulating many of the human disease symptoms, but subsequent rigorous analysis of all three mouse models have shown that the mice do not develop any behavioral, motor, or sensory deficits until $>12$ months of age, and these effects are fairly modest (Ganay et al., 2011). However, these mouse models do display IF aggregates that are a hallmark of the underlying disease pathology, including aggregations of neurofilaments, alpha-internexin, and peripherin in the central nervous system (CNS) that are visible as early as 3 months of age (Dequen et al., 2008).

In this experiment, we introduced the GFP or GAN gene into two of the GAN-KO mouse models $\left(\mathrm{GAN}^{\Delta e x 1}\right.$ and $\mathrm{GAN}^{\Delta e x 3-5}$ ) when these mice were 17-21 months old (see Supplementary Table S4 for a list of subjects and results summary). Under anesthesia, mice received a single injection into the cerebrospinal fluid of the cisterna magna. Ten microliters $\left(7.2 \times 10^{10}\right.$ total $\left.\mathrm{vg}\right)$ of AAV9/CMV-GAN was injected into $\mathrm{KO}$ mice or $10 \mu \mathrm{L}\left(8 \times 10^{10}\right.$ total vg) of AAV9/ CMV-GFP was injected into age-matched heterozygous mice, then the mice were allowed to recover. When injected into the cisterna magna in mice, AAV9 efficiently transduces large areas of the brainstem, dorsal spinal cord, motor neurons in the ventral horn, and to a much lesser extent other areas of the brain as visualized with GFP (Fig. 4A-C). In the absence of a good behavioral readout in these mice for a phenotypic rescue of function, a histological evaluation of IF aggregate resolution was pursued. Three weeks post-injection with the AAV9/CMV-GAN vector, mice were sacrificed for IHC evaluation of the IF aggregates in the brain. No IHC was done against gigaxonin because no suitable antibodies are available and IHC against the myc epitope failed. We observed nearly complete clearance of peripherin aggregates in the brainstem and dorsal lumbar spinal cord, in both the GAN $^{\Delta e x 1}$ and GAN ${ }^{\Delta e x 3-5} \mathrm{KO}$ mice (Fig. 4D-F, Supplementary Figs. S3 and S4). As expected, heterozygous mice did not show any abnormal peripherin staining and homozygous $\mathrm{KO}$ mice showed consistent and extensive aggregates of peripherin, at ages ranging from 8 to 24 months 
Table 1. Differentially Regulated Proteins Between GAN-Transfected and GFP-Transfected Gan Patients

\begin{tabular}{|c|c|c|c|c|c|c|c|}
\hline Acc. no. ${ }^{\mathrm{a}}$ & Protein name & $\%$ & $\mathrm{n}^{\mathrm{c}}$ & $P 1^{\mathrm{d}}$ & $P 2^{\mathrm{e}}$ & $P 3^{\mathrm{f}}$ & Localization \\
\hline O15145 & Actin-related protein $2 / 3$ complex subunit 3 & 19.1 & 6 & 1.29 & 1.61 & 1.33 & Cytoskeleton \\
\hline P60981 & Destrin $^{g}$ & 47.3 & 6 & 0.85 & 0.47 & 0.51 & Cytoskeleton \\
\hline Q14315 & Filamin- $\mathrm{C}^{\mathrm{g}}$ & 24 & 26 & 1.30 & 1.44 & 1.12 & Cytoskeleton \\
\hline P60660 & Myosin light polypeptide $6^{\mathrm{g}}$ & 66.9 & 11 & 0.77 & 0.83 & 0.61 & Cytoskeleton \\
\hline Q01995 & Transgelin ${ }^{\mathrm{g}}$ & 84.6 & 30 & 0.81 & 0.47 & 0.65 & Cytoskeleton \\
\hline O75347 & Tubulin-specific chaperone $\mathrm{A}^{\mathrm{g}}$ & 45.4 & 4 & 0.63 & 0.82 & 0.49 & Cytoskeleton \\
\hline P63104 & $14-3-3$ protein zeta/delta ${ }^{g}$ & 60 & 7 & 0.76 & 0.63 & 0.76 & Cytoplasm \\
\hline P15880 & $40 S$ ribosomal protein $S 2$ & 29.7 & 4 & 1.24 & 1.23 & 1.21 & Cytoplasm \\
\hline P36578 & $60 S$ ribosomal protein L4 & 42.9 & 10 & 1.18 & 1.28 & 1.54 & Cytoplasm \\
\hline P23588 & Eukaryotic translation initiation factor $4 \mathrm{~B}^{\mathrm{g}}$ & 27.3 & 6 & 0.55 & 0.80 & 0.72 & Cytoplasm \\
\hline Q15056 & Eukaryotic translation initiation factor $4 \mathrm{H}^{\mathrm{g}}$ & 16.1 & 4 & 0.52 & 0.80 & 0.61 & Perinuclear \\
\hline P09211 & Glutathione S-transferase $\mathrm{P}^{\mathrm{g}}$ & 30 & 10 & 0.74 & 0.67 & 0.89 & Cytoplasm \\
\hline O43795 & Myosin-Ib & 16.5 & 6 & 1.18 & 1.61 & 1.31 & Cytoplasm \\
\hline Q99497 & Protein DJ-1 ${ }^{g}$ & 32.8 & 7 & 0.74 & 0.62 & 0.65 & Cytoplasm \\
\hline P52565 & Rho GDP-dissociation inhibitor $1^{\mathrm{g}}$ & 43.1 & 5 & 0.75 & 0.41 & 0.66 & Cytoplasm \\
\hline P39656 & $\begin{array}{l}\text { Dolichyl-diphosphooligosaccharide-protein } \\
\text { glycosyltransferase 48-kDa subunitg }\end{array}$ & 15.7 & 5 & 1.48 & 1.66 & 1.70 & ER \\
\hline P04843 & $\begin{array}{l}\text { Dolichyl-diphosphooligosaccharide-protein } \\
\text { glycosyltransferase subunit } 1^{\mathrm{g}}\end{array}$ & 12.4 & 6 & 1.57 & 1.79 & 1.26 & ER \\
\hline O95302 & FK506-binding protein 9 & 20 & 7 & 1.37 & 1.24 & 1.15 & ER \\
\hline P55072 & Transitional endoplasmic reticulum ATPase ${ }^{g}$ & 41.2 & 20 & 1.42 & 1.25 & 1.17 & ER \\
\hline P23284 & Peptidyl-prolyl cis-trans isomerase $\mathrm{B}^{\mathrm{g}}$ & 68.5 & 18 & 1.45 & 1.52 & 1.17 & ER \\
\hline P09382 & Galectin-1 & 63 & 28 & 0.88 & 0.82 & 0.65 & Extracellular cytoplasm \\
\hline P15144 & Aminopeptidase $\mathrm{N}$ & 11.3 & 9 & 1.63 & 1.41 & 1.16 & Extracellular membrane \\
\hline P16070 & CD44 antigen ${ }^{\mathrm{g}}$ & 7.5 & 6 & 1.36 & 1.47 & 1.30 & Membrane \\
\hline Q00610 & Clathrin heavy chain $1^{\mathrm{g}}$ & 21.9 & 24 & 1.31 & 1.10 & 1.43 & Membrane \\
\hline Q9NZM1 & Myoferlin & 14.3 & 8 & 1.36 & 1.45 & 1.27 & Membrane \\
\hline O00469 & Procollagen-lysine,2-oxoglutarate 5-dioxygenase 2 & 17.5 & 4 & 1.41 & 1.50 & 1.33 & Membrane RER-membrane \\
\hline O60568 & Procollagen-lysine,2-oxoglutarate 5-dioxygenase 3 & 19.4 & 4 & 1.50 & 1.64 & 1.17 & Membrane RER- membrane \\
\hline Q6NUK1 & $\begin{array}{l}\text { Calcium-binding mitochondrial carrier } \\
\text { protein SCaMC-1 }\end{array}$ & 6.9 & 5 & 1.08 & 1.41 & 1.60 & $\begin{array}{l}\text { Mitochondrial inner } \\
\text { membrane }\end{array}$ \\
\hline Q9H1E3 & $\begin{array}{l}\text { Nuclear ubiquitous casein and cyclin-dependent } \\
\text { kinases substrate }\end{array}$ & 15.6 & 6 & 0.68 & 0.80 & 0.81 & Nucleus \\
\hline P22392 & Nucleoside diphosphate kinase $\mathrm{B}^{\mathrm{g}}$ & 50 & 7 & 0.89 & 0.71 & 0.74 & Nucleus \\
\hline Q9H299 & $\begin{array}{l}\text { SH3 domain-binding glutamic acid-rich-like } \\
\text { protein } 3^{\mathrm{g}}\end{array}$ & 53.8 & 5 & 0.67 & 0.84 & 0.77 & Nucleus \\
\hline
\end{tabular}

${ }^{\mathrm{a}}$ Uniprot accession number.

${ }^{b}$ Percentage of protein sequence coverage.

${ }^{\mathrm{C}}$ Number of peptides.

${ }^{\mathrm{d}}$ Patient 1 , ratio of GAN-transfected to GFP-transfected cells $(p<0.05)$.

ePatient 2, ratio of GAN-transfected to GFP-transfected cells $(p<0.05)$.

f Patient 3, ratio of GAN-transfected to GFP-transfected cells $(p<0.05)$.

groteins identified in this study but not altered between GAN patients and controls (Mussche et al., 2012).

(Supplementary Table S4). Expression of exogenous myctagged human gigaxonin in the brainstem was verified by Western blot analysis of brainstem tissue from treated mice, compared to heterozygous or untreated $\mathrm{KO}$ mice of similar age (Fig. 4G). The amount of gigaxonin in treated mice compared to heterozygous controls indicated a higher degree of overexpression in the mice at 3 weeks post-infection compared to the mild overexpression seen in fibroblasts at 3 days post-infection (compare WB in Fig. 4G to Fig. 2). Although the study investigated peripherin aggregates in agematched $\mathrm{KO}$ mice, and in some cases littermates, we wanted to verify to the best extent possible that the treated mice had IF aggregates pre-injection and that these were resolved as a result of the AAV9/GAN treatment. Because areas of the midbrain and forebrain were not efficiently targeted by AAV9 via this route of administration, they were stained for peripherin (midbrain, dorsal raphe nucleus) and neurofilament-heavy chain (NF-H, cortex and striatum) to investigate the presence of IF aggregates in these areas. Despite the absence of peripherin aggregates in the brainstem and dorsal spinal cord, the treated mice still had peripherin aggregates in the dorsal raphe nucleus and NF-H aggregates in the cortex and striatum (Supplementary Fig. S5). Although a comprehensive histopathological evaluation was not done on these mice, they did not display any adverse behavior phenotypes or gross brain pathology as a result of the treatment. These data provide strong evidence that the AAV9/GAN vector was capable of resolving long-standing peripherin IF aggregates in the CNS.

\section{Discussion}

As a whole, these experiments provide critical proof-ofconcept data that re-introduction of gigaxonin into affected $G A N$-null cells resolves IF aggregates, which are a major hallmark of the underlying disease pathology in mice and 


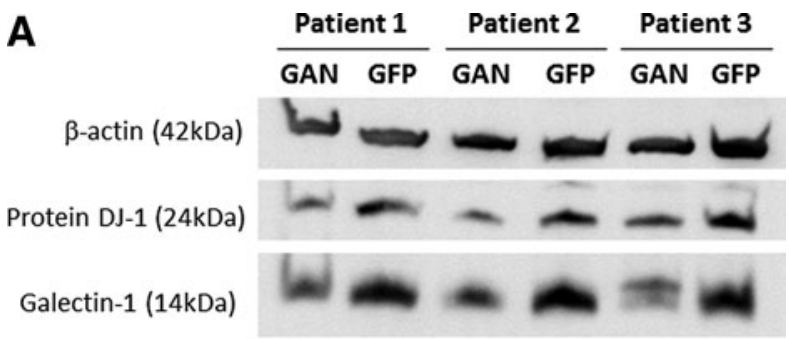

B

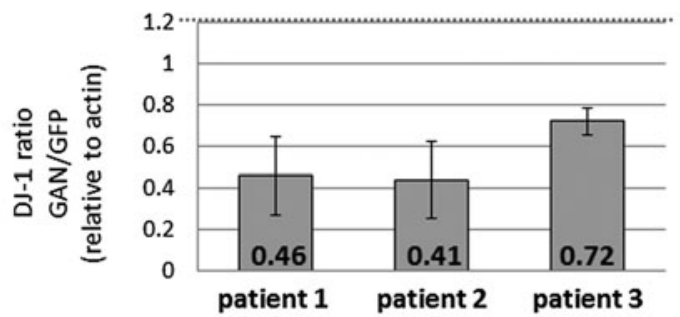

C

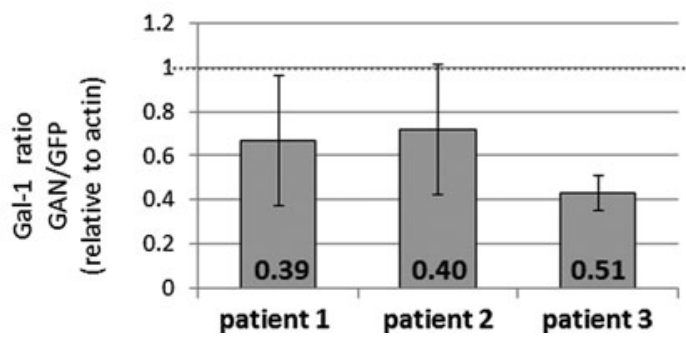

FIG. 3. Western blot validation of changes in protein levels. Western blot experiments were performed on protein extracts of fibroblasts after treatment with AAV vectors. (A) Representative Western blot analysis for proteins DJ-1 and galectin-1. (B, C) Densities of the bands were first measured and normalized against actin for each lane; values are the average from three Western blots. Then ratios were calculated by dividing the actin-normalized GAN value by the actin-normalized GFP value for each patient. A $p$ value was calculated based on the ratios of the three patients in the three Western blots. For both DJ-1 and galectin- 1 the changes were significant $(p<0.05)$. DJ-1 and galectin- 1 were downregulated in GAN-transduced cells compared to GFP-transduced cells, consistent with the iTRAQ proteomics results (Table 1). The validity of actin as a loading control was justified by its unchanged expression in GAN patients (Bomont et al., 2000; Pena et al., 1983). GAN=treated with AAV2/ CMV-GAN; GFP = treated with AAV2/CMV-GFP. Error bars are SD.

humans. This was demonstrated in vitro in cultured GANnull fibroblasts with the IF vimentin and in vivo in GAN-null mice with the IF peripherin. Moreover, the proteomic analysis of treated GAN primary fibroblasts provides important information with regards to more global changes (besides IFs) that occur in treated cells, which provides further insights into both the mechanism of the rescue and the basic biology of gigaxonin's function. While several reasonable conclusions can be drawn from these studies, the results should be considered within the appropriate context; namely that gigaxonin was overexpressed using the CMV promoter. Also, for the proteomic analysis, it is important to consider that this analysis was restricted to a single time point of 3 days post-infection. The timing was restricted to allow enough time for gigaxonin expression without prolonged maintenance of the dividing fibroblast culture (which would dilute transgene-expressing cells). Our results indicated that gigaxonin may play a role in multiple pathways affecting cytoskeleton regulation, and thus it may take longer than practical for an in vitro experiment to observe all proteomelevel changes, especially for proteins with long half-lives. Therefore we can only interpret the presence of proteomelevel changes, but not the absence of change.

\section{Proteome analysis}

Mussche et al. (2012) had previously done a proteomic analysis of GAN patient fibroblasts compared to normal control fibroblasts. One interesting finding is that three proteins that were previously found to be disregulated in GAN patients compared to healthy controls again showed altered expression levels in the AAV2/GAN-treated cells, only the regulation was seen in the other direction towards normal levels. This is the case for galectin-1, aminopeptidase $\mathrm{N}$ (APN), and FK506-binding protein 9. Interestingly, a set of proteins previously found to be differentially regulated in GAN patients versus controls was not found to be altered in response to the AAV2/GAN treatment (Supplementary Table S2). Those results would suggest that while some of the disregulated proteins in GAN can rapidly return to homeostatic levels in response to gigaxonin reintroduction, other changes may take longer to correct or may represent irreversible changes. However, since the proteomic changes in our experiment can be correlated to resolution of IF aggregates within the 3- to 4-day experimental timeframe, we can speculate that the proteins that modulated in response to gigaxonin reintroduction are important in the GAN disease mechanism. In particular a role for galectin-1 was previously proposed for GAN, as a regulator of cytoskeleton reorganization (Mussche et al., 2012). The FK506BP9 belongs to the FK506BP family, which comprises peptidyl prolyl isomerases that function as protein-folding chaperones and are also involved in the aggregation process of alpha-synuclein clusters in Parkinson disease (Gerard et al., 2012). APN is a transmembrane zinc-binding metallo-ectopeptidase that removes amino acids from unblocked $\mathrm{N}$-termini of bioactive peptides or proteins. It plays a role in cell migration and invasion of extracellular matrix, angiogenesis, and regulation of extrinsic regulatory factors of tumor growth, all conditions in which remodeling of the cytoskeleton is required. The introduction of the correct GAN-gene will activate several processes in reaction to this new gene, which is reflected in up- or downregulation of a set of proteins (Table 1). The majority of the differentially regulated proteins in the present study were previously identified in the proteomic analysis done by Mussche et al., but were not found to be disregulated in GAN fibroblasts (indicated in Table 1; Mussche et al., 2012). This may indicate that they are associated with GAN overexpression, rather than a return to a normal state. Some of these proteins were found to be associated with several ubiquitin specific peptidases (Sowa et al., 2009), and the ubiquitin-proteasome system, as listed in Supplementary Table S1. Ubiquitin-specific peptidases are a class of deubiquitinating enzymes that help to regulate many cellular processes by controlling the amount of the protein ubiquitin, through removing ubiquitin from a large variety of substrates (D'Andrea and Pellman, 1998; Wilkinson, 2000). But 

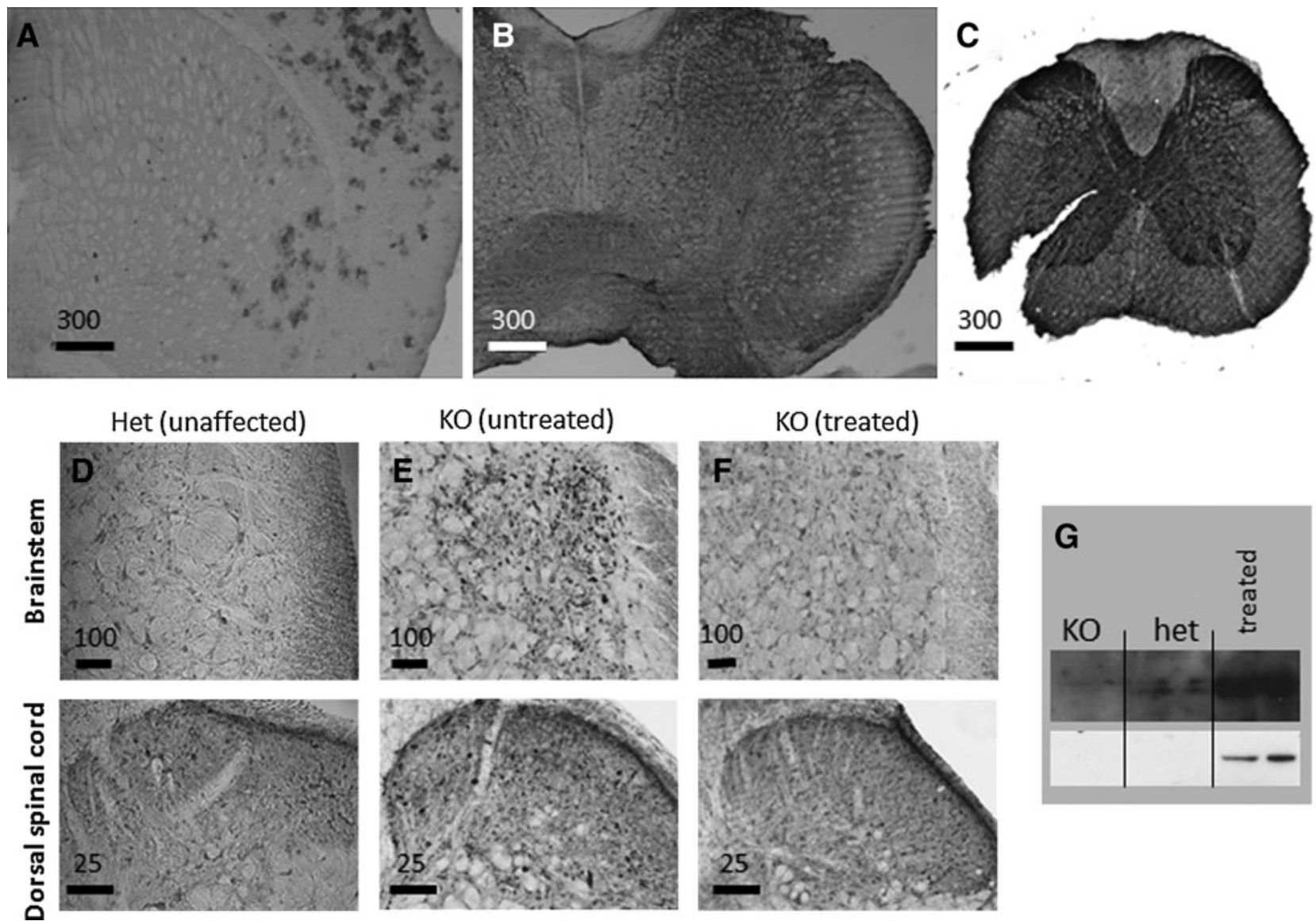

FIG. 4. Resolution of peripherin aggregates in GAN mice. Wild-type (WT) C57Bl/6J mice were injected with $8 \times 10^{10}$ vector genomes (vg) ssAAV9/CMV-GFP into the cerebral spinal fluid (CSF) of the cisterna magna, then GFP expression was assessed in the forebrain (A), brainstem (B), and lumbar spinal cord (C) by immunohistochemistry (IHC) against GFP. (C-F) The 17- to 21 -month-old GAN ${ }^{\Delta e x 1}(n=2)$ or GAN ${ }^{\Delta e x 3-5}(n=2)$ mice were injected with $7.2 \times 10^{10}$ vg of ssAAV2/CMV-GANv2 vector into the CSF of the cisterna magna. Three weeks post-injection the mice were sacrificed and sections of their brain and spinal cord assayed for peripherin accumulations by IHC. Results shown are indicative of all mice per group (see Supplementary Table S4 for a summary of all mice examined). Columns D-F: top panel shows the spinal trigeminal nucleus of the brainstem and the bottom set of panels show the dorsal lumbar spinal cord. Column D shows the IHC against peripherin in a normal mouse. Column E shows the pattern in a knock-out (KO) GAN mouse, and Column F shows mice of the same age treated with the vector. (G) Western blot brainstem tissue from a KO GAN mouse (KO, showing no gigaxonin), a heterozygous GAN mouse showing the low level of endogenous gigaxonin expression, and a treated KO GAN mouse expressing the myc-tagged gigaxonin transgene. The top row of bands are blotted with an anti-gigaxonin antibody, and the bottom row is a blot done in parallel with an anti-myc tag antibody. Samples are run in duplicate. Scale bars in panels A-F indicate micrometers. Larger annotated images are available as Supplementary Data.

targets, interactions, and specific functions of deubiquitinating enzymes are poorly understood. However, it is possible that in response to GAN overexpression they play a compensatory role by deubiquitination of targeted proteins.

Since GAN fibroblasts don't display a rise in the overall abundance of vimentin (Mussche et al., 2012) and there was no change in overall vimentin levels following AAV2/GAN treatment (Supplementary Table S3), we can speculate that the absence (and re-introduction) of gigaxonin is affecting the cytoskeletal organization of vimentin, rather than its total intracellular levels. The reduction of vimentin aggregates seen in transduced GAN fibroblasts indicates that there is a fairly rapid reorganization of the cytoskeleton. Several disregulated proteins were found associated with cytoskeleton reorganization (Table 1 ). Actin-related protein $2 / 3$ complex subunit 3 functions as a component of the Arp2/3 complex, which is involved in the regulation of actin polymerization and, together with an activating nucleation-promoting factor, mediates the formation of new branched actin networks. This is important during centrosome re-orientation before cytokinesis and in cytoskeletal organization. Destrin is an actin-depolymerizing protein, which severs actin filaments (F-actin) and binds to actin monomers (G-actin) and profilin4. Transgelin is an actin-crosslinking protein found in fibroblasts and muscle. The abundance of cytoskeleton proteins that were found changed in response to gigaxonin re-expression in this study reinforces gigaxonin's role in cytoskeleton organization, but a full model for all the interactions and pathways still remains elusive.

Another pertinent observation is the up-regulation of several endoplasmic reticulum resident proteins in GANtreated fibroblasts, in particular enzymes involved in protein 
synthesis, maturation, and post-translational modification, in addition to enzymes involved in endocytosis and recycling. This reflects a general return to a functional state of the fibroblast.

\section{Potential for GAN gene therapy}

GAN is a progressively fatal sensory and motor neuropathy. It begins with the loss of distal motor and sensory function, and as the disease progresses into the teenage years individuals will gradually lose the ability to walk and use their arms. The disease is normally fatal in the third decade of life, often due to respiratory dysfunction or other complications normally associated with a motor neuron disease. While GAN patients will display IF cytoskeletal disorganization in many cell types in their body, successful treatment of the spinal cord and brainstem would be hypothesized to treat the most serious disease phenotypes.

AAV9 vector administration into the vein, lumbar intrathecal space, and cisterna magna have all been validated as ways to achieve broad CNS transgene delivery in large animal models (Duque et al., 2009; Federici et al., 2012; Gray et al., 2013). In the present study, cisterna magna injections were modeled in mice to investigate a potential therapeutic approach for GAN that might be translatable to humans. Although the behavioral phenotype of these mice is very mild and therefore not amenable to assess a phenotypic rescue, they do display extensive IF aggregates. Encouragingly, there was widespread resolution of peripherin aggregates throughout the brainstem and spinal cord at 3 weeks following vector delivery. Considering that IF accumulations (including peripherin) are present as early as 3 months of age in GAN mice (Dequen et al., 2008), this result demonstrates the ability of the gigaxonin gene to resolve long-standing IF aggregates that are a hallmark of the disease pathology in vivo.

While the present studies implicate gigaxonin for a role in cytoskeleton organization, future studies are still needed to understand the exact cellular pathways affected by gigaxonin. In terms of a future gene therapy for GAN, the present study indicates the potential of gigaxonin gene replacement as a therapeutic approach for GAN. However, the use of a strong promoter and a single-stranded AAV genome is not ideal. Since gigaxonin is normally expressed at very low levels (Cleveland et al., 2009), overexpressionrelated toxicity is a concern and our studies suggest this possibility (see indicated changes in Table 1 and Supplementary Fig. S1), so a weak promoter may be sufficient and preferred. Self-complementary (sc)AAV vectors would greatly increase the transduction efficiency with AAV (Gray et al., 2011b; McCarty et al., 2003), which could be critical for human translation, although the 1.9-kb size of the gigaxonin gene may be challenging to fit in a $\sim 2.2-\mathrm{kb}$ scAAV genome with the appropriate transcriptional control elements. We speculate that these improvements would play an important role in the translation of these findings to future human application.

\section{Acknowledgments}

We wish to acknowledge funding from Hannah's Hope Fund. We are grateful for Yanmin Yang and Jean-Pierre Julien for providing the GAN mouse models. We thank
Huijing Sun for performing some of the intracisternal injections, and Haiyan Fu for showing us how to do them.

\section{Author Disclosure Statement}

The authors do not declare any conflicts of interest.

\section{References}

Allen, E., Ding, J., Wang, W., et al. (2005). Gigaxonin-controlled degradation of MAP1B light chain is critical to neuronal survival. Nature 438, 224-228.

Berg, B.O., Rosenberg, S.H., and Asbury, A.K. (1972). Giant axonal neuropathy. Pediatrics 49, 894-899.

Bomont, P., and Koenig, M. (2003). Intermediate filament aggregation in fibroblasts of giant axonal neuropathy patients is aggravated in non dividing cells and by microtubule destabilization. Hum. Mol. Genet. 12, 813-822.

Bomont, P., Cavalier, L., Blondeau, F., et al. (2000). The gene encoding gigaxonin, a new member of the cytoskeletal BTB/ kelch repeat family, is mutated in giant axonal neuropathy. Nat. Genet. 26, 370-374.

Bomont, P., Ioos, C., Yalcinkaya, C., et al. (2003). Identification of seven novel mutations in the GAN gene. Hum. Mutat. 21, 446.

Bruno, C., Bertini, E., Federico, A., et al. (2004). Clinical and molecular findings in patients with giant axonal neuropathy (GAN). Neurology 62, 13-16.

Cleveland, D.W., Yamanaka, K., and Bomont, P. (2009). Gigaxonin controls vimentin organization through a tubulin chaperone-independent pathway. Hum. Mol. Genet. 18, 13841394.

D'Andrea, A., and Pellman, D. (1998). Deubiquitinating enzymes: a new class of biological regulators. Crit Rev. Biochem. Mol. Biol. 33, 337-352.

Demir, E., Bomont, P., Erdem S, et al. (2005). Giant axonal neuropathy: clinical and genetic study in six cases. J. Neurol. Neurosurg. Psychiatry 76, 825-832.

Dequen, F., Bomont, P., Gowing, G., et al. (2008). Modest loss of peripheral axons, muscle atrophy and formation of brain inclusions in mice with targeted deletion of gigaxonin exon 1. J Neurochem. 107, 253-264.

Ding, J., Liu, J.J., Kowal, A.S., et al. (2002). Microtubuleassociated protein 1B: a neuronal binding partner for gigaxonin. J. Cell Biol. 158, 427-433.

Ding, J., Allen, E., Wang, W., et al. (2006). Gene targeting of GAN in mouse causes a toxic accumulation of microtubuleassociated protein 8 and impaired retrograde axonal transport. Hum. Mol. Genet. 15, 1451-1463.

Duque, S., Joussemet, B., Riviere, C., et al. (2009). Intravenous administration of self-complementary AAV9 enables transgene delivery to adult motor neurons. Mol. Ther. 17, 11871196.

Federici, T., Taub, J.S., Baum, G.R., et al. (2012). Robust spinal motor neuron transduction following intrathecal delivery of AAV9 in pigs. Gene Ther. 19, 852-859.

Furukawa, M., He, Y.J., Borchers, C., Xiong, Y. (2003). Targeting of protein ubiquitination by BTB-Cullin 3-Roc1 ubiquitin ligases. Nat. Cell Biol. 5, 1001-1007.

Ganay, T., Boizot, A., Burrer, R., et al. (2011). Sensory-motor deficits and neurofilament disorganization in gigaxonin-null mice. Mol. Neurodegener. 6, 25.

Gerard, M., Deleersnijder, A., Daniëls, V., et al. (2010). Inhibition of FK506 binding proteins reduces alpha-synuclein aggregation and Parkinson's disease-like pathology. J. Neurosci. 30, 2454-2463. 
Gray, S.J., Blake, B.L., Criswell, H.E., et al. (2010). Directed evolution of a novel adeno-associated virus (AAV) vector that crosses the seizure-compromised blood-brain barrier (BBB). Mol. Ther. 18, 570-578.

Gray, S.J., Choi, V.W., Asokan, A., et al. (2011a). Production of recombinant adeno-associated viral vectors and use in in vitro and in vivo administration. Curr. Protoc. Neurosci. Chapter 4: Unit 4.17.

Gray, S.J., Matagne, V., Bachaboina, L., et al. (2011b). Preclinical differences of intravascular AAV9 delivery to neurons and glia: a comparative study of adult mice and nonhuman primates. Mol. Ther. 19, 1058-1069.

Gray, S.J., Nagabhushan Kalburgi, S., McCown, T.J., and Jude Samulski, R. (2013). AAV9 transduction in the central nervous system of non-human primates. Hum. Gene Ther. doi: 10.1038/gt.2012.101.

Houlden, H., Groves, M., Miedzybrodzka, Z., et al. (2007). New mutations, genotype phenotype studies and manifesting carriers in giant axonal neuropathy. J. Neurol. Neurosurg. Psychiatry 78, 1267-1270.

Koop, O., Schirmacher, A., Nelis, E., et al. (2007). Genotypephenotype analysis in patients with giant axonal neuropathy (GAN). Neuromuscul. Disord. 17, 624-630.

Kuhlenbäumer, G., Young, P., Oberwittler, C., et al. (2002). Giant axonal neuropathy (GAN): case report and two novel mutations in the gigaxonin gene. Neurology 58, 1273-1276.

Leung, C.L., Pang, Y., Shu, C., et al. (2007). Alterations in lipid metabolism gene expression and abnormal lipid accumulation in fibroblast explants from giant axonal neuropathy patients. BMC Genet. 8, 6.

McCarty, D.M., Fu, H., Monahan, P.E., et al. (2003). Adenoassociated virus terminal repeat (TR) mutant generates selfcomplementary vectors to overcome the rate-limiting step to transduction in vivo. Gene Ther. 10, 2112-2118.

Mussche, S., De Paepe, B., Smet, J., et al. (2012). Proteomic analysis in giant axonal neuropathy: New insights into disease mechanisms. Muscle Nerve doi: 10.1002/mus.23306.

Nalini, A., Gayathri, N., Yasha, T.C., et al. (2008). Clinical, pathological and molecular findings in two siblings with giant axonal neuropathy (GAN): report from India. Eur. J. Med. Genet. 51, 426-435.

Pena, S.D., Opas, M., Turksen, K., et al. (1983). Immunocytochemical studies of intermediate filament aggregates and their relationship to microtubules in cultured skin fibroblasts from patients with giant axonal neuropathy. Eur. J. Cell Biol. 31, 227-234.
Perkins, D.N., Pappin, D.J., Creasy, D.M., and Cottrell, J.S. (1999). Probability-based protein identification by searching sequence databases using mass spectrometry data. Electrophoresis 20, 3551-3567.

Prineas, J.W., Ouvrier, R.A., Wright, R.G., et al. (1976). Giant axonal neuropathy-generalized disorder of cytoplasmic microfilament formation. J. Neuropathol. Exp. Neurol. 35, 458-470.

Reidegeld, K.A., Eisenacher, M., Kohl, M., et al. (2008). An easyto-use Decoy Database Builder software tool, implementing different decoy strategies for false discovery rate calculation in automated MS/MS protein identifications. Proteomics 8, 1129-1137.

Snel, B., Lehmann, G., Bork, P., and Huynen, M.A. (2000). STRING: a web-server to retrieve and display the repeatedly occurring neighbourhood of a gene. Nucleic Acids Res. 2000. 28, 3442-3444.

Sowa, M.E., Bennett, E.J., Gygi, S.P., and Harper, J.W. (2009). Defining the human deubiquitinating enzyme interaction landscape. Cell 138, 389-403.

Wang, W., Ding, J., Allen, E., et al. (2005). Gigaxonin interacts with tubulin folding cofactor $\mathrm{B}$ and controls its degradation through the ubiquitin-proteasome pathway. Curr. Biol. 15, 2050-2055.

Wilkinson, K.D. (2000). Ubiquitination and deubiquitination: targeting of proteins for degradation by the proteasome. Semin. Cell Develop. Biol. 11, 141-148.

Zhang, D.D., Lo, S.C., Sun, Z., et al. (2005). Ubiquitination of Keap1, a BTB-Kelch substrate adaptor protein for Cul3, targets Keap1 for degradation by a proteasome-independent pathway. J. Biol. Chem. 280, 30091-30099.

Address correspondence to: Dr. Steven J. Gray Gene Therapy Center

Thurston-Bowles Bldg, Room 7109 University of North Carolina Chapel Hill, NC 27599-7352

E-mail: graysj@email.unc.edu

Received for publication May 30, 2012; accepted after revision December 31, 2012.

Published online: January 14, 2013. 